\title{
Modeling of the propagation of Bessel beams in a uniaxial crystal at different positions of the crystal axis
}

\author{
Krasnov A.P.
}

Samara State Aerospace University

\begin{abstract}
The numerical study of the propagation of the Bessel beams in anisotropic media with different orientations of the crystal axis and different polarizations of the input beams has been carried out using distributed computing on supercomputers. The analysis is based on two models: the geometric optics model based on ray tracing and implemented using the ZEMAX software, and the wave model in the approximation of thin optical elements based on plane waves expansion and implemented using the VectorAnisotropicPropagators package. High-performance computing resources have been necessary to implement the wave model. The results obtained can be used in various fields of optical design, for example to determine the position of the crystal axis and for the development of devices that perform polarization conversion.
\end{abstract}

Keywords: Bessel beams, axicon, astigmatic transformations, uniaxial crystal, polarization

Citation: Krasnov A.P. Modeling of the propagation of Bessel beams in a uniaxial crystal at different positions of the crystal axis. Proceedings of Information Technology and Nanotechnology (ITNT-2015), CEUR Workshop Proceedings, 2015; 1490: 69-81. DOI: 10.18287/1613-0073-2015-1490-69-81

\section{Introduction}

One of the major challenges for optical information science is the calculation of the propagation of electromagnetic waves in different media. Thus, optical devices that allow certain properties of electromagnetic radiation to be converted have acquired growing interest and practical use. Most often, mode and polarization conversions are needed. One tool for such transformations are anisotropic crystals [120]. One method of modelling the propagation of electromagnetic waves in anisotropic media is the method of plane waves expansion [21-26]. As a rule, the use of this method causes large computational problems due to the need to calculate the direct and inverse Fourier transforms for all electromagnetic field components. One solution is the use of various fast algorithms, including FFT, which significantly reduces computation time. However, the use of the FFT algorithm has drawbacks associated with the fixed discreteness of input and output signals and the ability to calculate only the transverse distribution. Another option is the use of parallel algorithms using high performance computing resources (supercomputers). 
In this work, a comparative modeling of the propagation of the Bessel and Gaussian beams with different polarization in a uniaxial crystal along and perpendicular to the axis of the crystal is carried out. It is shown that in this case there are different effects: one is the astigmatic distortion and the second is the interference interaction of ordinary and extraordinary rays. Furthermore, the influence of the anisotropic medium in the different types of modes has been varied and is dependent on the distribution of the spatial spectrum of the beam.

The simulation has been carried out in three different ways - ray tracing, plane waves expansion and the finite-difference time-domain method. In the last two cases parallel execution of programs on supercomputers is used. A comparison of methods for speed, efficiency, scalability and the nature of the results has been performed.

\section{The theoretical part}

The laser beam with a super-Gaussian amplitude is determined by the formula:

$\Psi_{m}(r, \varphi)=\exp \left(-\left(\frac{r}{\sigma}\right)^{p}\right) \exp (\operatorname{im} \varphi)$,

where $\sigma$ is the radius of the beam waist.

Selecting $p=2$ and $\varphi=0$ we get the usual Gaussian beam, which will be modelled in the future:

$$
F_{\sigma}(r)=\exp \left(-\frac{r^{2}}{\sigma^{2}}\right) \text {. }
$$

In order to observe the paraxial effects, the beam is focused through the converging lens. Instead of lenses, it is possible to use the multiplication of the input beam amplitude with the corresponding converging wave front:

$\exp \left(-\frac{i k r^{2}}{2 f}\right)$

where $f$ is the focal length of the lens.

Another laser beam selected for modelling is the Bessel mode:

$F_{m, \alpha}(r, \varphi, z)=$

$=A \cdot J_{m}(\alpha r) \cdot \exp (i m \varphi) \cdot \exp \left(i z \sqrt{k^{2}-\alpha^{2}}\right)$,

where $\alpha$ is the large-scale radial index of a Bessel beam and $m$ is the angular index.

Instead of the Bessel beam, it is acceptable to simulate the passage of a flat beam through a linear axicon:

$\exp (-i k p r)$,

where $k=2 \pi / \lambda$ is the wave number and $p=N A$ is the numerical aperture of the axicon.

The complex function of the axicon means that its structure is kept at a certain distance, but it has similar properties and does not require infinite energy. 


\section{Implementation}

The geometric optics approach is implemented in various commercial software products (e.g., ZEMAX, LightTrans) and is used to obtain a visual representation of the investigated phenomena.

The ZEMAX program has been used for optical simulation. A detailed description of the software used is given in the manual [27]. The important advantage of this software is the ability to examine the distribution of ordinary and extraordinary rays separately.

Submit the input Gaussian beam and place in front of the crystal thin converging lens. Then the ordinary and extraordinary rays will have different angles of refraction at the interface of two media, which will give rise to different effects.

Figures 1-2 show that when the crystal axis is parallel to the $z$ axis, the ordinary and extraordinary rays will focus at two different points on the $z$ axis. Thus in the general case the output beam has elliptical polarization, the shape of which at any given point depends on the transverse coordinates and is different for these rays.

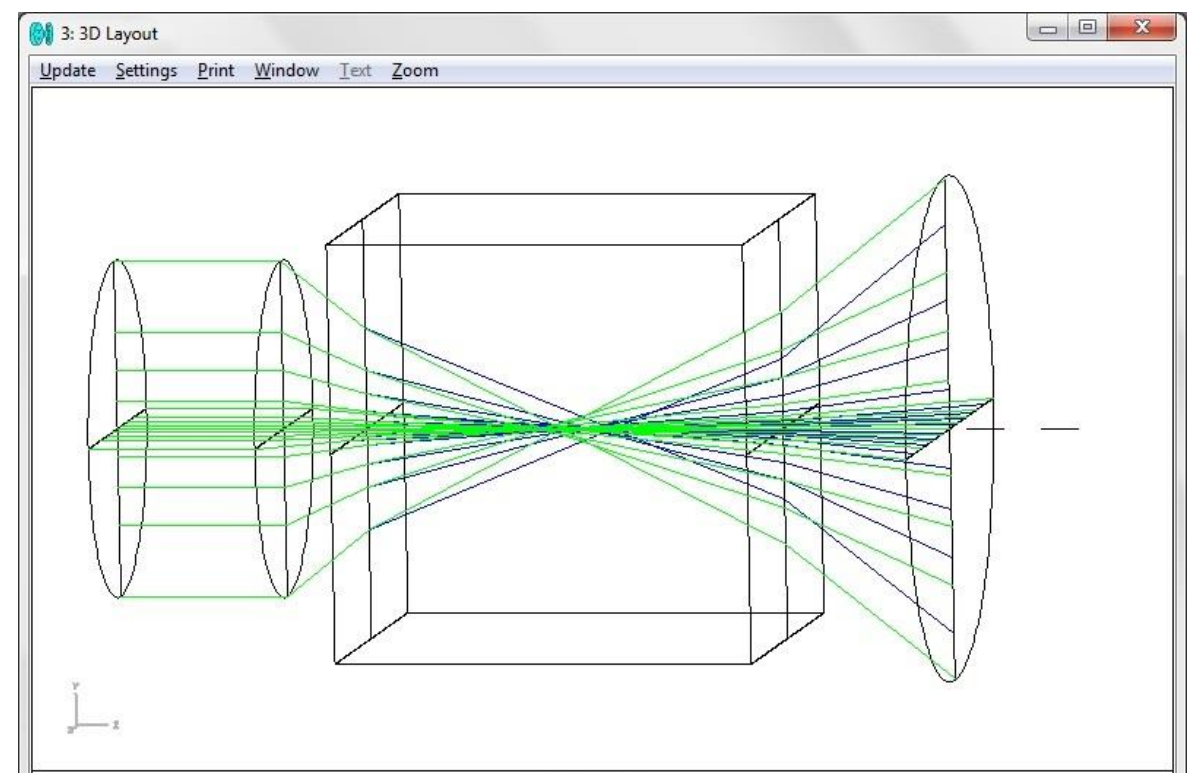

Fig. 1. - The focusing of the Gaussian beam transmitted through a lens in the crystal of an Icelandic spar at the location of the crystal axis parallel to the $z$ axis

We have applied a flat beam for the entrance and put the axicon in its way to simulate the Bessel beam as in the ZEMAX program it is not possible to specify an arbitrary type of input beam. 

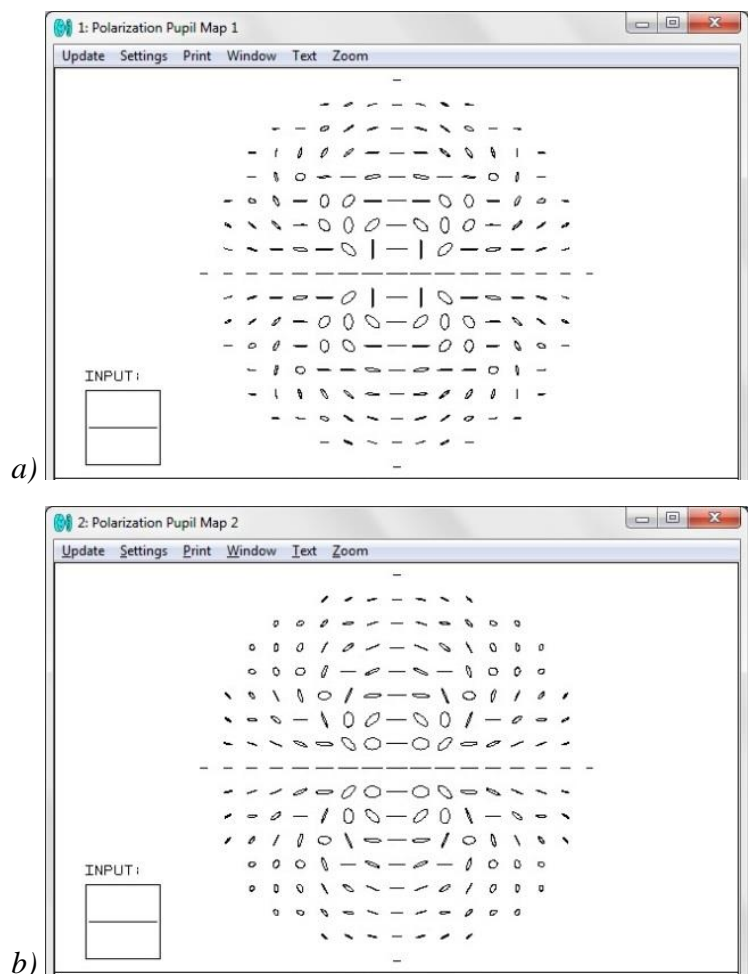

Fig. 2. - The polarization of the Gaussian beam transmitted through a lens at the exit of the crystal of an Icelandic spar: a - the ordinary ray, b - the extraordinary ray

Figures 3-4 show the simulation results of beam propagation in the crystal of an Icelandic spar along the axis of the crystal. It is evident that the ordinary and extraordinary rays are focused in different ways, however the result of their interaction remains unclear. During propagation along the crystal axis, the beam is elliptically polarized. For ordinary ray, polarization is close to linear and varies in a complicated way depending on the transverse coordinates, whereas for extraordinary ray polarization ellipses in an elongated fashion along the initial polarization of the beam.

The use of ray tracing does not require considerable investment of time and allows for the focus in the crystal at different positions of the axis to be explored and for the beam polarization to be changed. However, it is difficult to define wave characteristics of generated fields in this case. Therefore, we have considered the focusing of radiation in an anisotropic medium on the basis of the wave theory of diffraction [21-26]. 


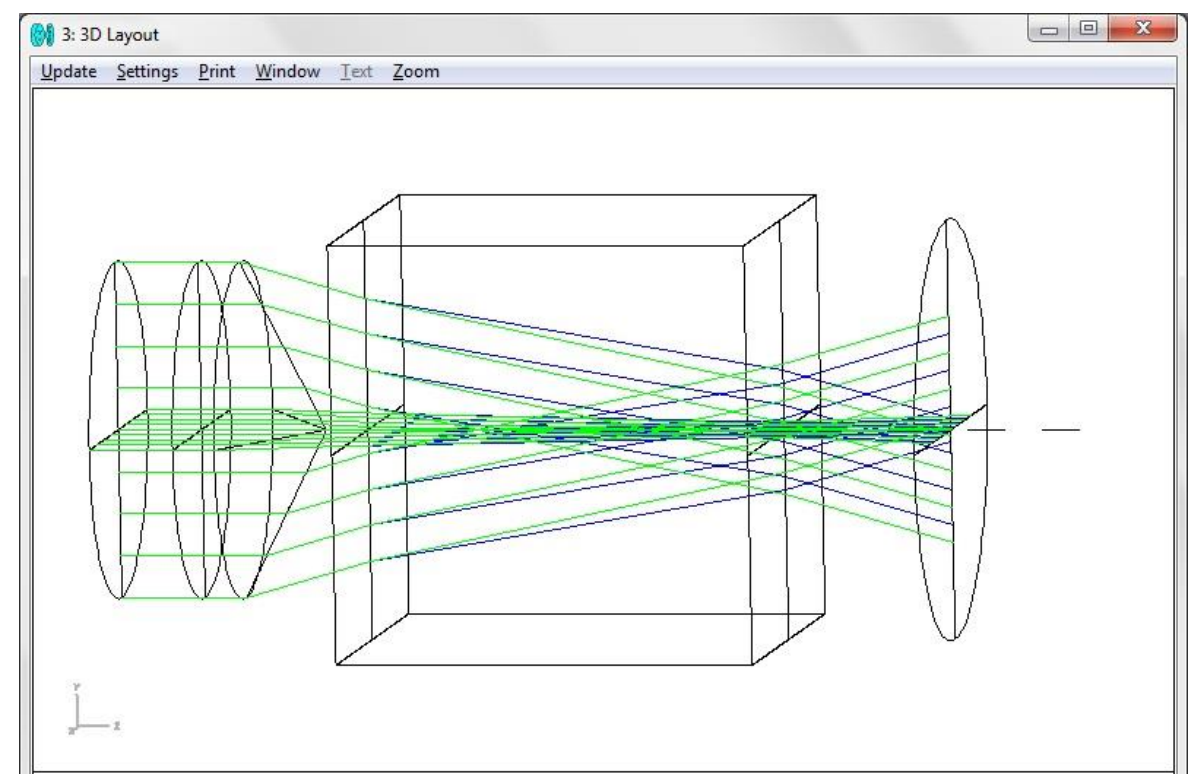

Fig. 3. - The focusing of the flat beam having passed through an axicon in the crystal of an Icelandic spar at the location of the crystal axis parallel to the $\mathrm{z}$ axis
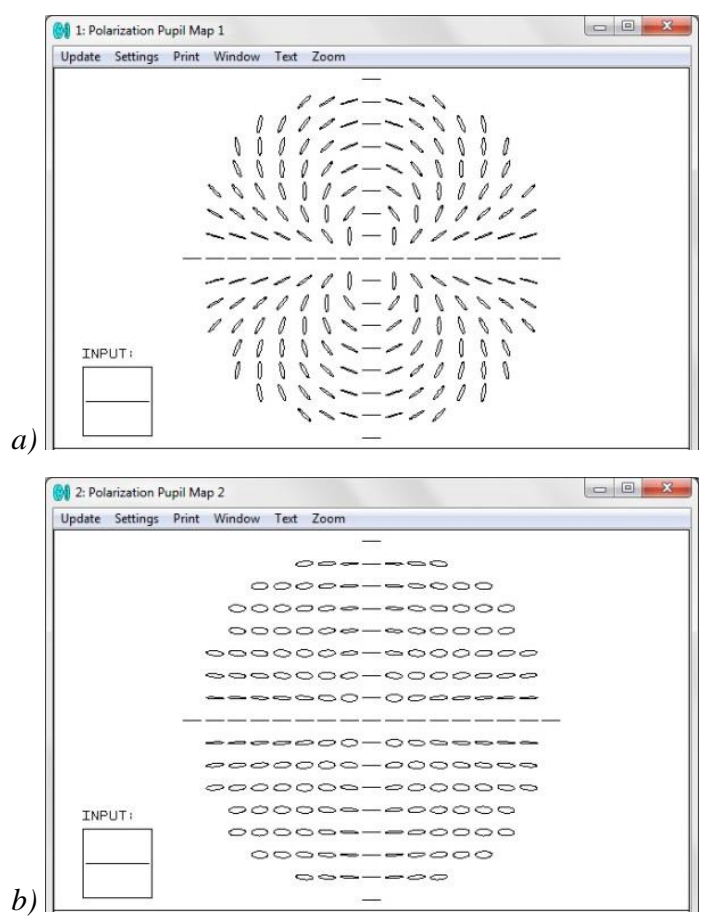

Fig. 4. - The polarization of the flat beam having passed through an axicon, at the exit of the crystal of an Icelandic spar: a - the ordinary ray, b - the extraordinary ray 


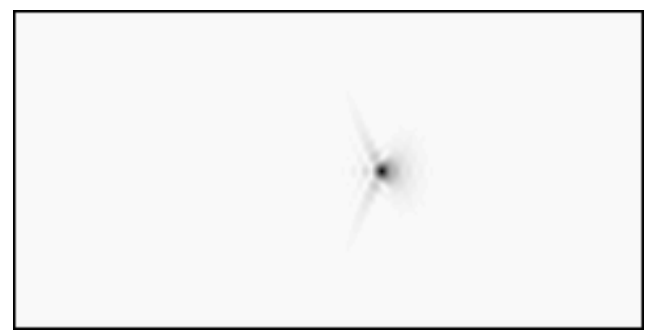

Fig. 5. - The longitudinal section of the amplitude of the electric field during $x$-polarization of the beam in the axial plane, $z=\overline{0,1500} \mu m$, the axis of the crystal is oriented along the $y$ axis

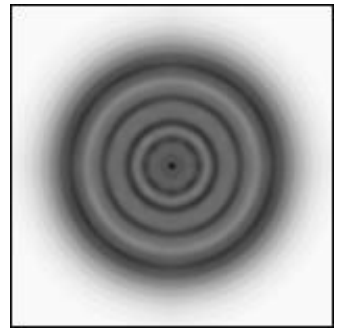

Fig. 6. - The cross section of the amplitude of the electric field during x-polarization of the beam in the plane $z=750 \mu m$, the axis of the crystal is oriented along the $y$ axis

The input Gaussian beam is submitted with a converging wave front corresponding to the thin lens in the path of the beam.

The passage of the beam in an Icelandic spar is considered.

The first case is the axis of the crystal is oriented along the y axis.

Following this, the passage of the $\mathrm{x}$-polarized beam is simulated. Figure 5 shows a longitudinal section of the amplitude, and figure 6 is the cross section of the amplitude near the area of focus of the beam. It is evident that the beam is distributed identically to an isotropic medium that is without a division into ordinary and extraordinary rays and without distortion.

Polarization is defined at the angle of 45 degrees to the $x$ axis. At this point, the split of the beam into ordinary and extraordinary rays appears: the former is unchanged while the second undergoes a distortion, stretching itself along the axis of the crystal. Figures 7 and 8 confirm this by showing the longitudinal and cross section of the beam as a whole and for each of the rays. 


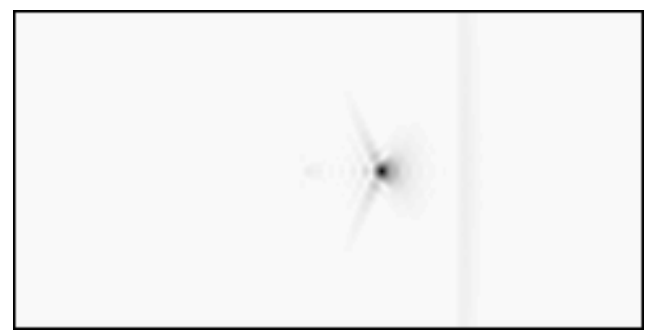

Fig. 7. - The longitudinal section of the amplitude of the electric field during $x y$-polarization of the beam in the axial plane, $z=\overline{0,1500} \mu \mathrm{m}$, the axis of the crystal is oriented along the $y$ axis

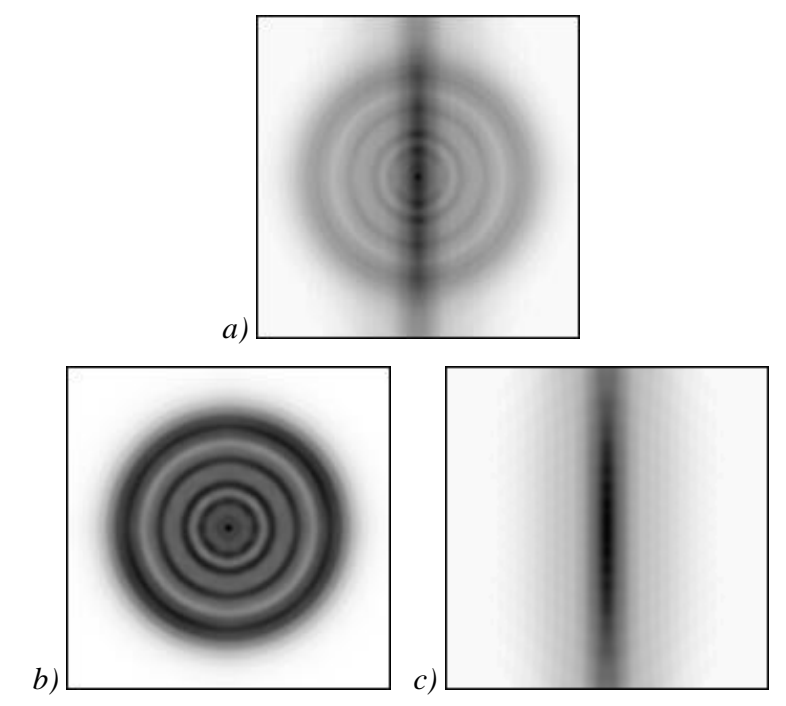

Fig. 8. - The cross section of the amplitude of the electric field during $x y$-polarization of the beam in the plane $z=750 \mu \mathrm{m}$, the axis of the crystal is oriented along the $y$ axis: a - the total amplitude, $\mathrm{b}$ - the ordinary ray, $\mathrm{c}$ - the extraordinary ray

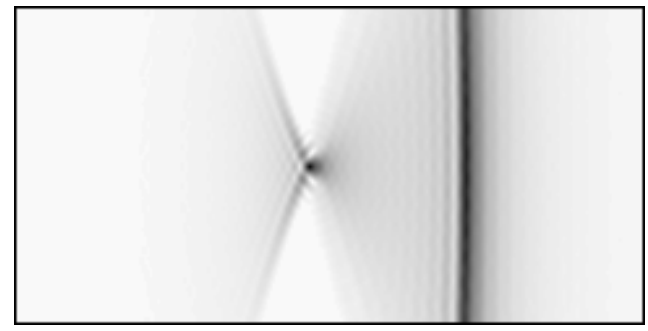

Fig. 9. - The longitudinal section of the amplitude of the electric field during $y$-polarization of the beam in the axial plane, $z=\overline{0,1500} \mu \mathrm{m}$, the axis of the crystal is oriented along the $y$ axis 


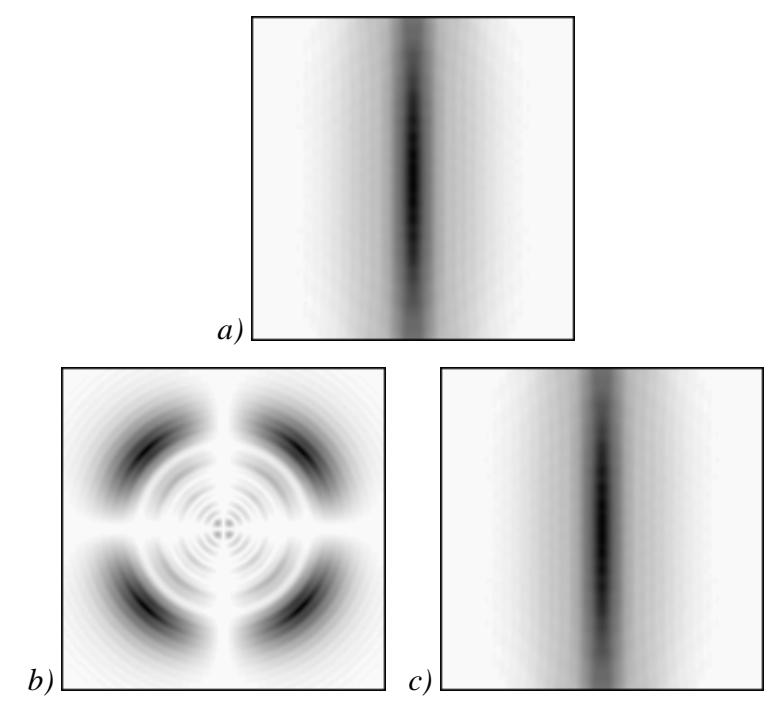

Fig. 10. - The cross section of the amplitude of the electric field during $y$-polarization of the beam in the plane $z=750 \mu \mathrm{m}$, the axis of the crystal is oriented along the $y$ axis: a - the total amplitude, $\mathrm{b}$ - the ordinary ray, $\mathrm{c}$ - the extraordinary ray

The input beam is defined as y-polarized. Here, the electric field is formed mainly by the extraordinary ray, which is elongated as in the previous case, and the contribution of the ordinary ray is negligible (figures 9-10).

The second case is where the crystal axis is oriented along the $\mathrm{z}$ axis.

Where the linear polarization of the beam is defined the two focuses are formed on the optical axis, the near focus is formed by the extraordinary ray and the far focus is formed by the ordinary ray. This is well illustrated in figures 11-12.

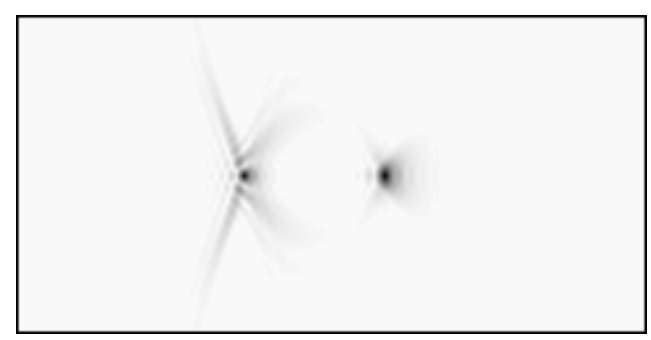

Fig. 11. - The longitudinal section of the amplitude of the electric field in the axial plane, $z=\overline{0,1500} \mu \mathrm{m}$, the axis of the crystal is oriented along the $z$ axis 


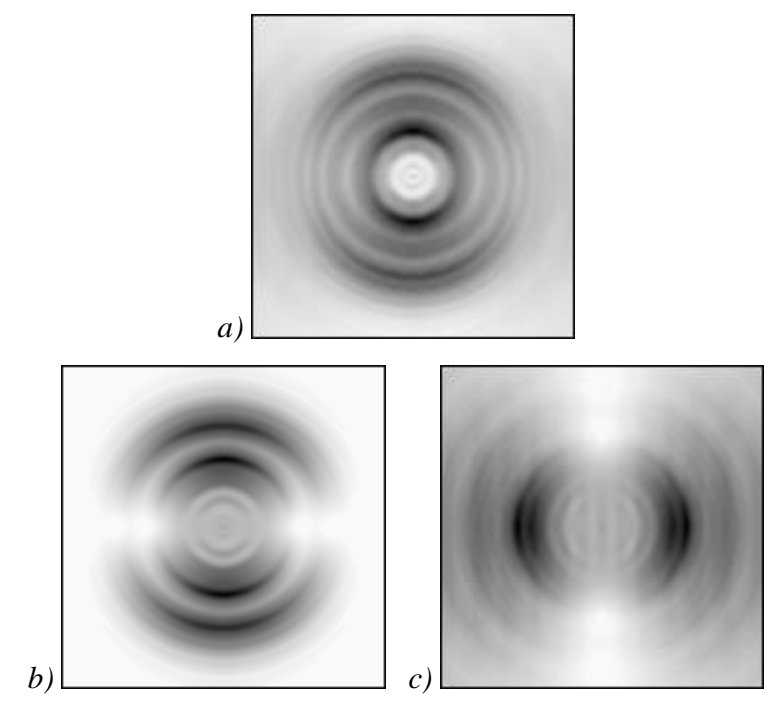

Fig. 12. - The cross section of the amplitude of the electric field in the plane $z=750 \mu m$, the axis of the crystal is oriented along the $z$ axis: $\mathrm{a}$ - the total amplitude, $\mathrm{b}$ - the ordinary ray, $\mathrm{c}-$ the extraordinary ray

The Bessel beam has also been considered.

In the case where the crystal axis is oriented along the y axis and the beam has an y-polarization, the electric field is almost completely formed by the extraordinary ray, which is astigmatically distorted (figures 13-14).

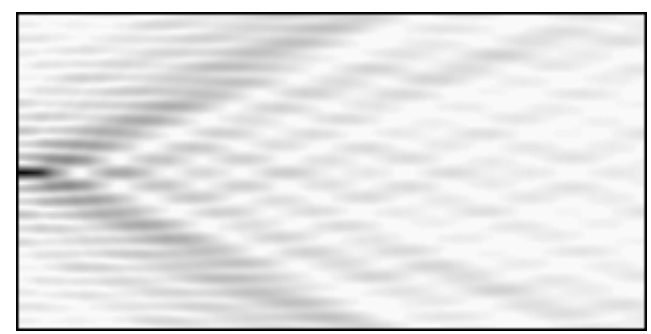

Fig. 13. - The longitudinal section of the amplitude of the electric field during y-polarization of the beam in the axial plane, $z=\overline{0,1500} \mu m$, the axis of the crystal is oriented along the $y$ axis 

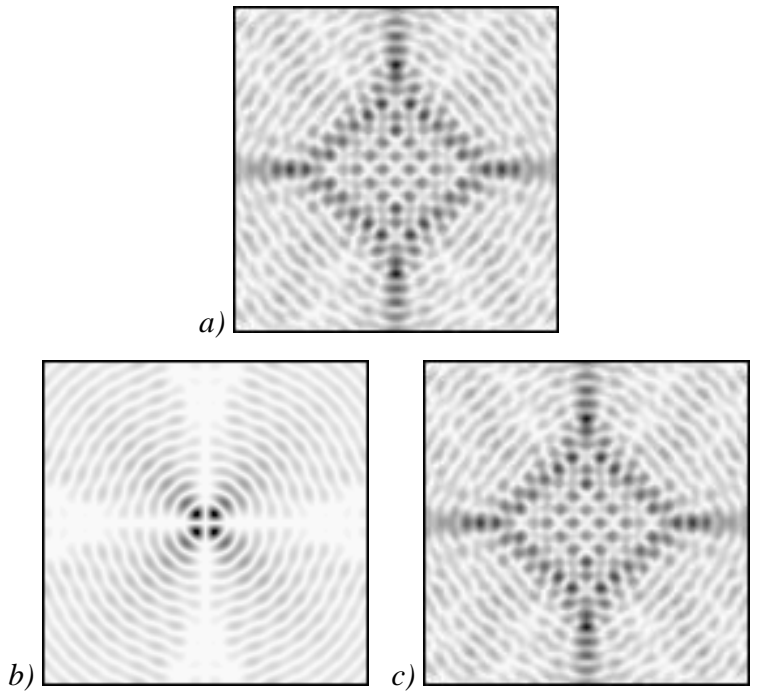

Fig. 14. - The cross section of the amplitude of the electric field during $y$-polarization of the beam in the plane $z=250 \mu \mathrm{m}$, the axis of the crystal oriented along the $y$ axis: a - the total amplitude, $\mathrm{b}$ - the ordinary ray, $\mathrm{c}$ - the extraordinary ray

At the x-polarization the beam is distributed as an isotropic medium, which means there is no distortion and the structure is preserved to certain distance.

The most interesting results have been obtained when modelling the propagation of the Bessel beam along the crystal axis. Due to the interference of the ordinary and extraordinary rays, there are periodic oscillations of the axial intensity that persisted until the collapse of the beam that is clearly visible in figures 15-16.

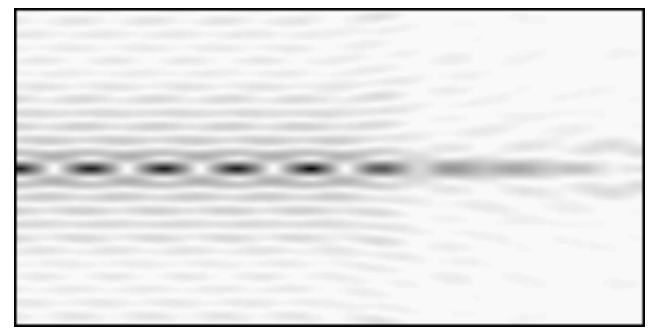

Fig. 15. - The longitudinal section of the amplitude of the electric field in the axial plane, $z=\overline{0,1500} \mu \mathrm{m}$, the axis of the crystal is oriented along the $z$ axis 


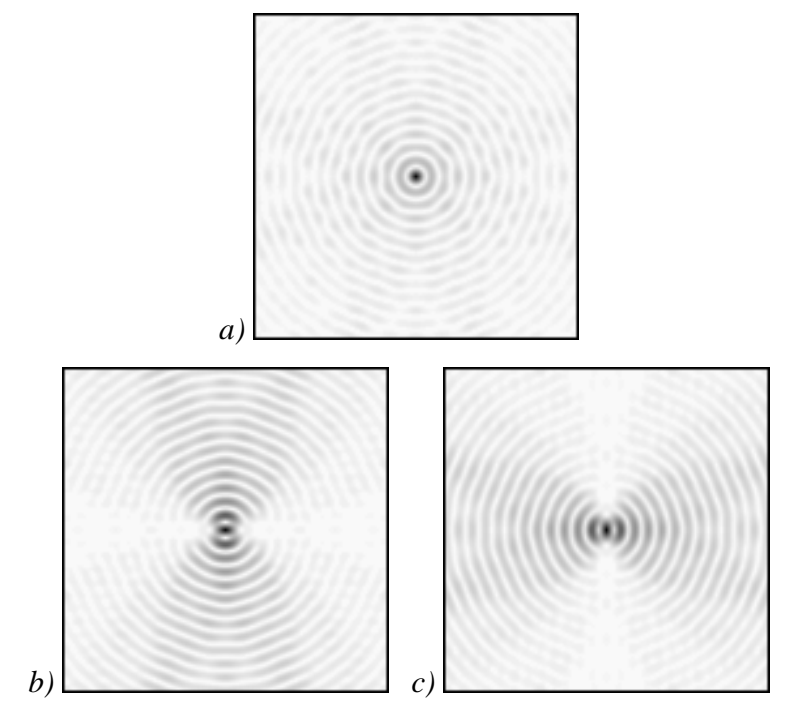

Fig. 16. - The cross section of the amplitude of the electric field in the plane $z=500 \mu m$, the axis of the crystal is oriented along the $z$ axis: $\mathrm{a}$ - the total amplitude, $\mathrm{b}$ - the ordinary ray, $\mathrm{c}-$ the extraordinary ray

\section{Conclusion}

The following results have been obtained in the course of work.

Numerical simulations of the propagation of Gaussian and Bessel beams in a birefringent medium have been conducted. It has been shown that under certain conditions there is spatial separation of the ordinary and extraordinary rays; the more extensive the path the beam passes in the crystal, the stronger the discrepancy.

The two beams are formed through the focusing of a Gaussian beam in the crystal having a different wave front curvature due to the variation in the refractive indices of the ordinary and extraordinary rays.

At the propagation in the crystal, the axis of which is perpendicular to the propagation direction and the polarization direction, the Bessel beam behaves identically as in a homogeneous medium maintaining the mode properties. Otherwise, the Bessel beam experiences an astigmatic distortion. At the propagation of a Bessel beam along the axis of the crystal, in addition to the astigmatic transformation, there is also a periodic variation in the intensity on the axis of propagation; this is associated with the interference of the ordinary and extraordinary rays.

The study was carried out in several ways, including using high-performance computing facilities, which allows physical processes to be visualized. The dependence of the time of the programs work on different parameters is demonstrated. It is easy to verify that the various simulation methods give qualitatively consistent results.

Geometric optics modelling does not require considerable investment of time and allows the visualization of the polarization transformation to be implemented conveniently as the focusing in the crystal well at different positions of the axis and 
the changes in the beam polarization are tracked. However, it is difficult to define the wave characteristics of the generated fields in this case.

Wave simulation based on the decomposition of flat waves makes it possible to quantify the characteristics of the generated electromagnetic fields, but does not take into account the physical properties of the elements making up the bundles and requires a lot of time for the calculation.

A numerical simulation with a different type of beam polarization and different positions of the crystal axis allows the conditions under which there is the greatest astigmatic distortion of the beams to be determined. The analysis can be useful in practice for ascertaining the position of the crystal axis.

\section{Acknowledgements}

This work was financially supported by the Russian Ministry of Education and Science.

\section{References}

1. Fedorov FI. Optics of anisotropic media. Minsk: Publishing House of the Academy of Sciences of the BSSR, 1958. 381 p. [in Russian]

2. Yariv A, Yeh P. Optical waves in crystals. New York: Wiley, 1984. 589 p.

3. Khilo NA, Petrova ES, Ryzevich AA. Conversion order Bessel beams in uniaxial crystals. Quantum Electronics, 2001, 31(1): 85-89.

4. Ciattoni A, Cincotti G, Palma C. Propagation of cylindrically symmetric fields in uniaxial crystals. Journal of the Optical Society of America A, 2002; 19(4): 792-796.

5. Hacyan S, Jáuregui R. Evolution of optical phase and polarization vortices in birefringent media. Journal of Optics A: Pure and Applied Optics, 2009; 11(8): 085204-085208.

6. Zusin DH, Maksimenka R, Filippov VV, Chulkov RV, Perdrix M, Gobert O, Grabtchikov AS. Bessel beam transformation by anisotropic crystals. Journal of the Optical Society of America A, 2010; 27(8): 1828-1833.

7. Fadeyeva TA, Shvedov VG, Izdebskaya YV, Volyar AV, Brasselet E, Neshev DN, Desyatnikov AS, Krolikowski W, Kivshar YS. Spatially engineered polarization states and optical vortices in uniaxial crystals. Optics Express, 2010; 18(10): 10848-10863.

8. Khonina SN, Volotovsky SG, Kharitonov SI. Periodic intensity change for laser mode beams propagating in anisotropic uniaxial crystals. News of the Samara Scientific Center of RAS, 2012; 14(4): 18-27.

9. Khonina SN, Zoteeva OV, Kharitonov SI. Nonparaxial propagation of Gaussian beams at an angle to the axis of an anisotropic crystal. Computer Optics, 2012; 36(3): 346-356.

10. Khilo NA. Diffraction and order conversion of Bessel beams in uniaxial crystals. Optics Communications, 2012; 285(5): 503-509.

11. Zhou G, Chen R, Chu X. Propagation of Airy beams in uniaxial crystals orthogonal to the optical axis. Optics Express, 2012; 20(3): 2196-2205.

12. Zhou Y, Wang X, Dai C, Chu X, Zhou G. Nonparaxial analysis in the propagation of a cylindrical vector Laguerre-Gaussian beam in a uniaxial crystal orthogonal to the optical axis. Optics Communications, 2013; 305: 113-125.

13. Zoteeva OV, Khonina SN. Diffraction of Bessel laser beams on a birefringent object. Proc of SPIE, 2013; 9156: 91560D-8. 
14. Khonina SN, Volotovsky SG, Kharitonov SI. Features of nonparaxial propagation of Gaussian and Bessel beams along the axis of the crystal. Computer Optics, 2013; 37(3): 297-306.

15. Li J, Chen Y, Cao Q. Propagation properties of cylindrically polarized vector beam through uniaxial crystals along the optical axis. Optics \& Laser Technology, 2013; 45: 364-372.

16. Khonina SN, Morozov AA, Karpeev SV. Effective transformation of a zero-order Bessel beam into a second-order vortex beam using a uniaxial crystal. Laser Physics, 2014; 24(5): 056101-056105.

17. Krasnov AP, Khonina SN. Comparative modelling of laser beam propagation in a uniaxial crystal based on integral operators. Bulletin of Samara State Aerospace University, 2014; 43(1): 238-252.

18. Khonina SN, Kharitonov SI. Comparative investigation of nonparaxial mode propagation along the axis of uniaxial crystal. Journal of Modern Optics, 2015; 62(2): 125-134.

19. Turpin A, Loiko YV, Kalkandjiev TK, Mompart J. Light propagation in biaxial crystals. Journal of Optics, 2015; 17: 065603-065606.

20. Khonina SN, Karpeev SV, Alferov SV, Soifer VA. Generation of cylindrical vector beams of high orders using uniaxial crystals. Journal of Optics, 2015; 17: 065001-065011.

21. Born M, Wolf E. Principles of optics. Cambridge: Cambridge University Press, 1999. 986 p.

22. Luneburg RK. Mathematical Theory of Optics. Berkeley. California: University of California Press, 1964. $440 \mathrm{p}$

23. Fleck JA Jr, Feit MD. Beam propagation in uniaxial anisotropic media. Journal of the Optical Society of America, 1983; 73(7): 920-926.

24. Ciattoni A, Crosignani B, Di Porto P. Vectorial theory of propagation in uniaxially anisotropic media. Journal of the Optical Society of America A, 2001; 18(7): 1656-1661.

25. Doskolovich LL, Kazanskiy NL, Kharitonov SI. Integral representations of solutions of a system of Maxwell's equations for anisotropic media. Computer Optics, 2010; 34(1): 5257

26. Khonina SN, Kharitonov SI. An analog of the Rayleigh-Sommerfeld integral for anisotropic and gyrotropic media. Journal of Modern Optics, 2013; 60(10): 814-822.

27. ZEMAX. Optical Design Program. User's Guide. ZEMAX Development Corporation 2009. $766 \mathrm{p}$. 